\title{
Traitement préventif antirabique humain post-exposition et animaux terrestres enragés en Ontario, au Canada : de 2014 à 2016
}

\author{
Middleton ${ }^{1 *}$, L Friedman ${ }^{1}$, S Johnson ${ }^{1}$, S Buchann ${ }^{1}$, B Warshawsky ${ }^{1}$
}

\section{Résumé}

Contexte : Le nombre d'animaux terrestres enragés en Ontario affiche une forte baisse depuis les années 1970 et 1980. Cependant, le nombre de traitements préventifs antirabiques post-exposition (TPAPE) recommandés n'a pas diminué de manière proportionnelle. La décision de recommander un TPAPE lors d'une exposition à des animaux terrestres doit se fonder sur une évaluation du risque qui tient compte de la prévalence de la rage chez ces animaux au sein d'un territoire, entre autres facteurs.

Objectif : Explorer les tendances en matière de recommandations de TPAPE vis-à-vis de I'exposition à des animaux terrestres en Ontario par rapport la date du dernier cas de rage chez les animaux terrestres par territoire des bureaux de santé publique (BSP).

Méthodologie : On a obtenu les données concernant les recommandations de TPAPE de 36 BSP de l'Ontario du système d'information en santé publique intégré ontarien et les données de rage animale par BSP du ministère des Richesses naturelles et des Forêts. Nous avons calculé les taux annuels de recommandation de TPAPE pour les animaux terrestres par BSP pour 2014 à 2016, puis mis en plan les taux de 2016 par rapport à l'année de la plus récente identification d'animal terrestre enragé au sein du BSP.

Résultats : Entre 2014 et 2016, les taux annuels de recommandation de TPAPE pour I'exposition à des animaux terrestres par BSP allaient de 3,0 à 35,2 par 100000 personnes, avec une médiane de 11,9 recommandations de TPAPE par 100000 personnes. En 2016, 10 BSP n'avaient pas identifié d'animal terrestre enragé dans leur compétence depuis plus de 15 ans. Cinq de ces BSP présentaient des taux de recommandation de TPAPE supérieurs à la médiane provinciale.

Conclusion : Conjuguée à d'autres facteurs, la considération de la présence de rage chez des animaux terrestres dans un territoire peut simplifier l'évaluation du risque concernant les chiens, chats ou furets qui ne sont pas accessibles pour fin d'observation ultérieure.
Citation proposée : Middleton D, Friedman L, Johnson S, Buchan S, Warshawsky B. Traitement préventif antirabique humain post-exposition et animaux terrestres enragés en Ontario, au Canada : de 2014 à 2016. Relevé des maladies transmissibles au Canada. 2019;45(7/8):196-202. https://doi.org/10.14745/ccdr.v45i78a02f Mots-clés : traitement préventif antirabique post-exposition, rage animale, évaluation du risque de rage
Cette oeuvre est mise à la disposition selon les termes de la licence internationale Creative Commons Attribution 4.0

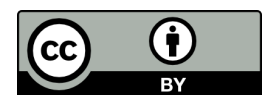

Affiliation

1 Santé publique Ontario, Toronto, Ontario

${ }^{\star}$ Correspondance :

dean.middleton@oahpp.ca

\section{Introduction}

On n'a pas décelé de cas de rage chez l'humain découlant d'une exposition à un animal terrestre depuis plus de 50 ans au Canada (communication personnelle, B. Stevenson, ministère des Richesses naturelles et des Forêts de l'Ontario, 22 août 2017). Quoi qu'il en soit, étant donné l'effet mortel presque universel de l'infection rabique après l'apparition des symptômes, la rage provenant d'un animal terrestre continue de constituer une préoccupation de santé publique. En Ontario, au Canada, le nombre d'animaux terrestres identifiés comme souffrant de la rage s'est avéré très bas au cours des dernières années (figure 1), avec deux exceptions. Ces exceptions étaient une épizootie de la rage du raton laveur dans le centre-ouest de l'Ontario, qu'on a identifiée à la fin de 2015 et qui provenait d'un raton laveur transféré, ainsi qu'une enzootie continue de 
la rage du renard arctique dans le sud-ouest et le centreouest de l'Ontario (1). On attribue la baisse marquée de la rage chez les animaux terrestres en Ontario au programme de vaccination des animaux sauvages contre la rage du ministère des Richesses naturelles et des Forêts de l'Ontario, qui a commencé en 1989. En tant que mesure supplémentaire visant à prévenir les cas de rage humaine en Ontario, le traitement préventif antirabique post-exposition (TPAPE) est financé par l'État et facilement accessible (2).

Figure 1 : Cas de rage animale par type d'animal : Ontario, 2006 à 2017

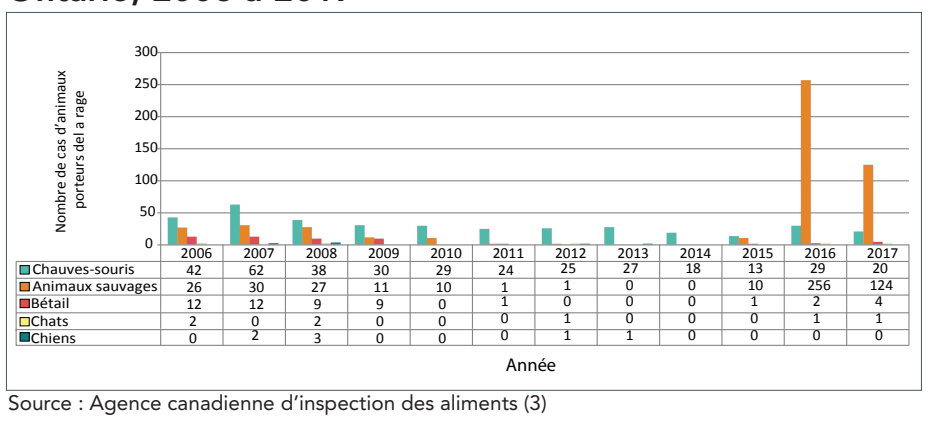

Malgré la rareté des cas de rage humaine en Ontario et la baisse marquée du nombre d'animaux terrestres enragés, le nombre annuel de TPAPE recommandés n'a pas diminué de manière proportionnelle (4-6) (figure 2). Administrer un TPAPE lorsque ce n'est pas indiqué entraîne un risque d'événement indésirable et de coût sans présenter d'avantage. Une étude, dans le contexte d'une très faible incidence de cas de rage animale, a permis de calculer que le risque de mortalité découlant d'un accident d'automobile pendant le trajet pour aller recevoir le TPAPE est supérieur au risque de mortalité découlant de la rage (7).

Conformément au Protocole de prévention et de contrôle de la rage de l'Ontario, on devrait fonder la décision de recommander le TPAPE lors d'une exposition à des animaux sur une évaluation du risque qui tient compte de nombreux facteurs tels que la prévalence de la rage chez ces animaux dans un territoire $(8,9)$. Le personnel des 35 bureaux de santé publique (BSP) d'Ontario (36 avant le $1^{\text {er }}$ mai 2018) effectue une évaluation du risque après l'exposition d'une personne à un animal pouvant être porteur de la rage, bien qu'au bout du compte, le fournisseur de soins de santé décide de recommander le TPAPE ou non. Lorsqu'un chien, un chat ou un furet mord ou griffe une personne, le BSP met l'animal en observation pendant 10 jours. Si l'animal est sain à la fin de la période post-exposition de 10 jours, on le considère comme incapable d'avoir transmis la rage au moment de l'exposition. Dans ce cas, le TPAPE n'est pas indiqué.

Avant 2013, les lignes directrices canadiennes et ontariennes recommandaient le TPAPE dans tous les cas lorsque le chien, le chat ou le furet n'était pas accessible pour une période d'observation de 10 jours. En 2013, on a actualisé les lignes directrices dans le but de recommander une évaluation du risque visant à déterminer si le TPAPE est indiqué après l'exposition à l'animal. Les éléments de l'évaluation du risque pour un animal terrestre qui n'est pas accessible aux fins d'observation comprennent le type d'exposition (c'est-à-dire morsure, absence de morsure), l'emplacement anatomique de l'exposition, le risque de rage chez l'espèce animale concernée, la présence de rage dans la région où l'incident s'est produit et les circonstances qui entourent l'exposition (c.-à-d. expositions avec motif ou

Figure 2 : Nombre annuel de cas de rage animale (animaux terrestres et chauves-souris) et nombre annuel de traitements préventifs antirabiques post-exposition administrés ou recommandés (y compris les expositions à l'extérieur de l'Ontario) ${ }^{\mathrm{b}}$, Ontario, 1958 à 2017

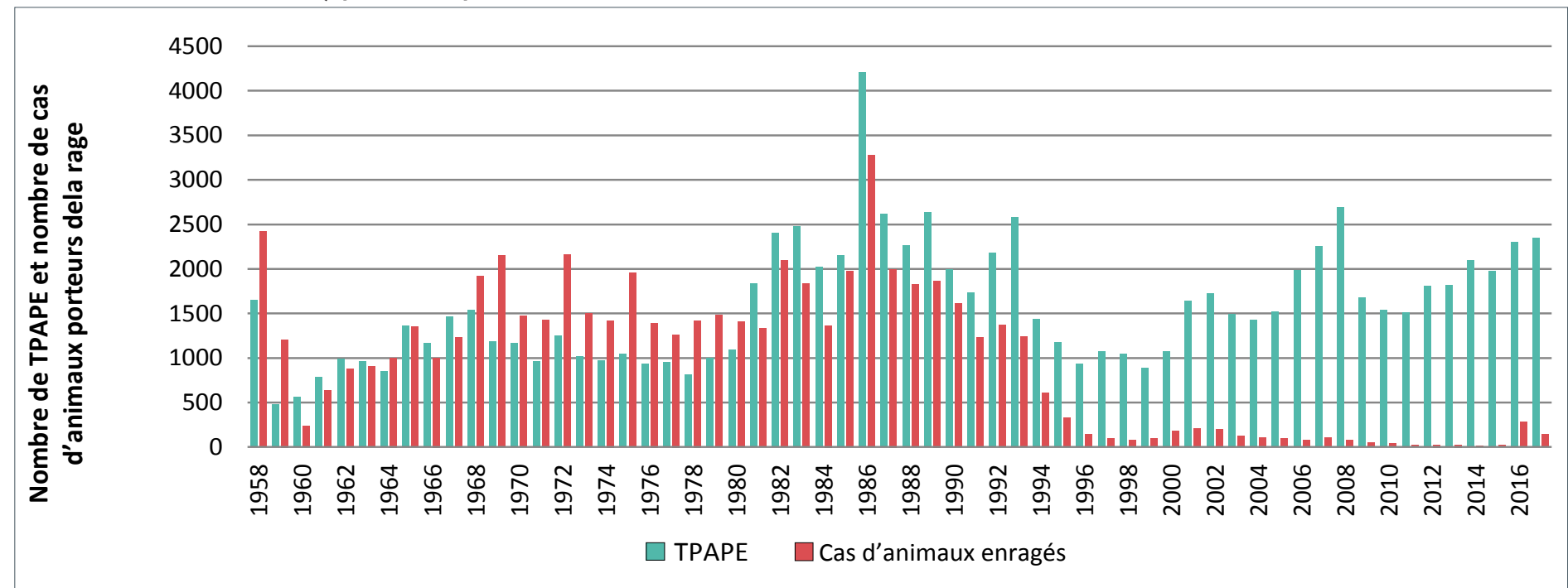

Abréviation : TPAPE, traitement préventif antirabique post-exposition

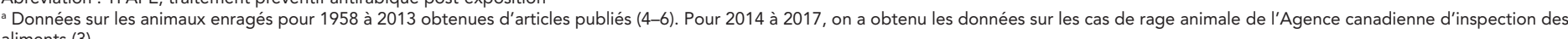
aliments (3)

b Données sur le TPAPE pour 1958 à 2013 obtenues d'articles publiés (4-6). Pour 2014 à 2016, on a extrait des données de TPAPE non nominatives du Système d'information sur la santé

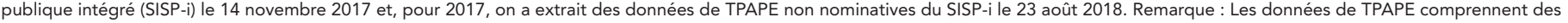
recommandations à la fois pour les animaux terrestres et les chauves-souris, qu'on ait administré le TPAPE ou non et que l'exposition se soit produite en Ontario ou ailleurs 
sans motif), ainsi que la fiabilité des antécédents de la personne blessée $(8,10,11)$.

Dans le présent article, nous explorons la relation entre les cas locaux de rage chez les animaux terrestres et le taux de recommandation de TPAPE par chaque BSP. Pour cette analyse, nous comparons les taux de recommandation de TPAPE de 2016 concernant l'exposition à des animaux terrestres pour chaque BSP avec l'année au cours de laquelle on a identifié le dernier animal terrestre enragé dans chaque compétence.

\section{Méthodologie}

\section{Sources des données}

Données sur le traitement préventif antirabique postexposition

En Ontario, les incidents avec exposition à un animal possiblement enragé pour lesquels le TPAPE est recommandé (peu importe s'il est administré ou non) sont saisis dans le Système d'information sur la santé publique intégré (SISP-i) par le BSP où le client réside (12). Dans le SISP-i, on demande aux BSP de remplir plusieurs champs avec les renseignements associés à l'incident, ce qui comprend de l'information sur la personne exposée, les circonstances entourant l'exposition, l'animal (s'il est accessible aux fins d'observation, sa situation de vaccination connue, etc.) et si le TPAPE est recommandé. Une partie de commentaires à texte libre permet d'ajouter de l'information supplémentaire sur le cas.

On a extrait les données de TPAPE du SISP-i de 2014 à 2016 le 14 novembre 2017. On a uniquement inclus les dossiers avec recommandations de TPAPE concernant les expositions à des animaux terrestres s'étant déroulées en Ontario dans les analyses. Ces dossiers comprenaient les expositions à des espèces ontariennes connues comme étant des réservoirs de rage (c.-à-d. ratons laveurs, renards et mouffettes) et des vecteurs passerelles (c.-à-d. chiens, chats et bétail, y compris bovins, chevaux, moutons, chèvres et lamas). On a exclu les dossiers avec une exposition s'étant déroulée à l'extérieur de l'Ontario et qui concernent des espèces animales qui ne sont pas terrestres, ou encore des espèces animales indiquées comme étant " inconnues", à moins que des renseignements dans la partie de commentaires $n$ 'indiquent que ces animaux soient terrestres et $n$ 'indiquent pas que l'exposition se soit produite à l'extérieur de l'Ontario.

\section{Données sur la population}

On a obtenu les estimations de la population pour chaque BSP de 2014 à 2016 de IntelliHEALTH Ontario $(13,14)$.

\section{Données sur les animaux}

Le ministère des Richesses naturelles et des Forêts a fourni des données sur l'année du dernier animal terrestre enragé confirmé signalé pour chaque BSP depuis 2016 (communication personnelle, B. Stevenson, ministère des Richesses naturelles et des Forêts, 29 juin 2017).

\section{Analyses}

On a calculé les taux de recommandation de TPAPE par BSP pour 2014 à 2016. Nous avons utilisé les données de TPAPE annuelles de chaque BSP concernant l'exposition à des animaux terrestres en tant que numérateur et l'estimation de la population pour ce BSP en tant que dénominateur, en plus d'illustrer les résultats par un graphique.

On a également calculé le nombre d'années depuis I'identification du dernier animal terrestre enragé dans chaque BSP comparativement à 2016. On a catégorisé les BSP dans un des cinq intervalles quinquennaux suivants conformément au plus récent signalement d'un animal terrestre enragé : 2016 ou les quatre années précédentes, les 6 à 10 années précédentes, les 11 à 15 années précédentes, et plus de 15 annéesprécédentes. Nous avons représenté dans un graphique les taux de recommandation de TPAPE de 2016 concernant l'exposition à des animaux terrestres par BSP, en classant chaque BSP par sa catégorie en ce qui concerne le nombre d'années depuis l'identification du dernier animal terrestre enragé dans ce BSP comparativement à 2016. On a utilisé le logiciel Excel de Microsoft [version 2010; Microsoft Corporation, Redmond, Washington, États-Unis (É.-U.)] pour analyser les données et produire les graphiques. On a réalisé la cartographie et la préparation des données spatiales pour ces données au moyen du logiciel des systèmes d'information géographique (SIG) ArcMap (version 10.3; ESRI, Redlands, Californie, É.-U.)

\section{Résultats}

Les taux annuels de recommandation de TPAPE concernant I'exposition à des animaux terrestres par BSP pour 2014 à 2016 allaient de 3,0 à 35,2 par 100000 personnes, avec une médiane de 11,9 recommandations de TPAPE par 100000 personnes. Les taux annuels de recommandation de TPAPE semblaient relativement stables, avec 10 BSP présentant des taux de TPAPE supérieurs à la médiane et 15 BSP présentant des taux inférieurs à la médiane, pour les trois années (figure 3).

En 2016, 10 BSP n'avaient pas identifié d'animal terrestre enragé dans leur territoire depuis plus de 15 ans (figure 4). Cinq de ces BSP présentaient des taux de recommandation de TPAPE supérieurs à la médiane provinciale. Deux autres BSP, dont aucun n'a identifié un animal terrestre enragé au cours des 11 à 15 années précédentes, présentaient également des taux de TPAPE supérieurs à la médiane (figure 5). 
Figure 3 : Taux annuels de recommandation de traitement préventif antirabique post-exposition concernant l'exposition à des animaux terrestres par bureau de santé publiquea, Ontario, 2014 à 2016

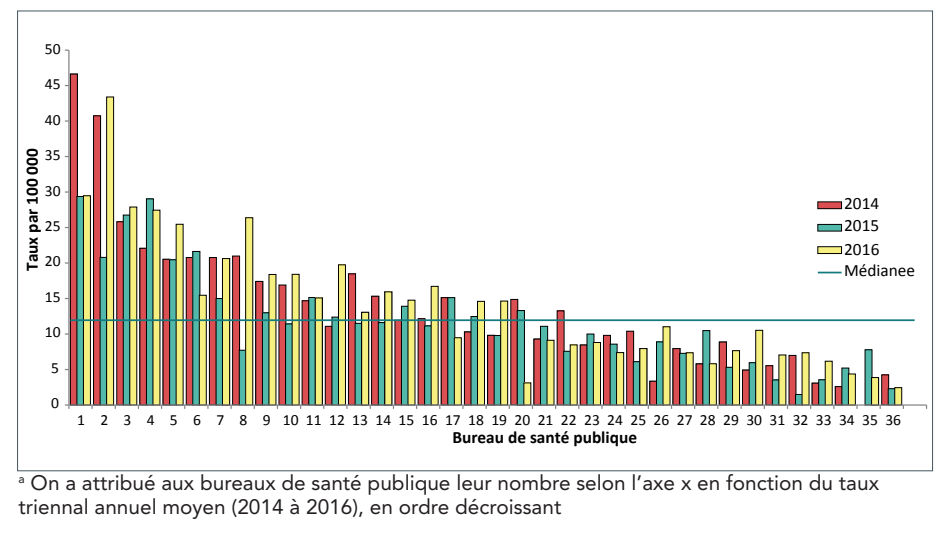

Figure 4 : Dernier cas confirmé de rage chez un animal terrestre par bureau de santé publique, Ontario

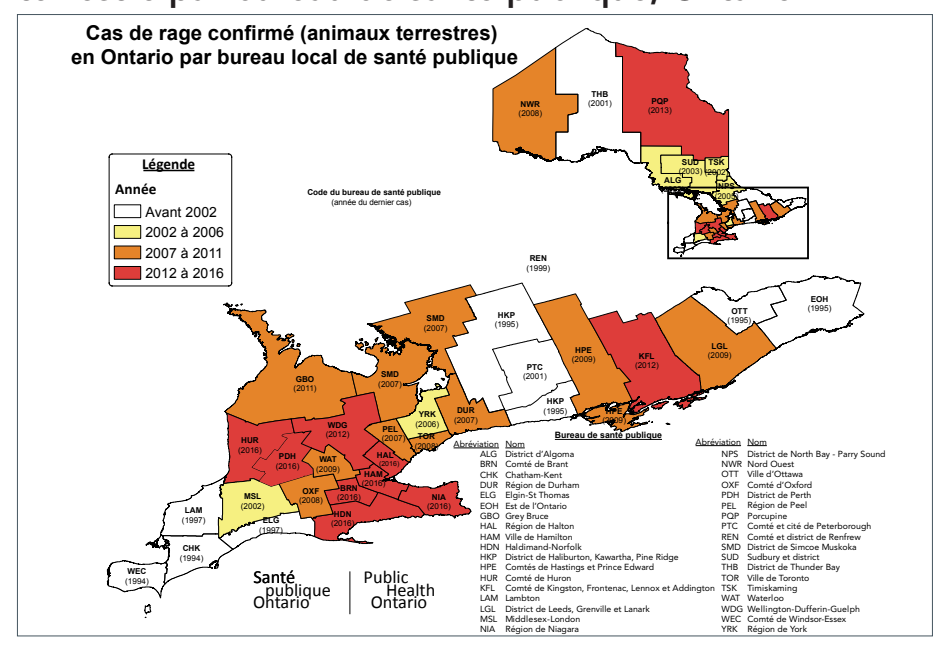

Figure 5 : Taux de recommandation de traitement préventif antirabique post-exposition concernant l'exposition à des animaux terrestres en Ontario par bureau de santé publique ${ }^{a}$ pour 2016 et nombre d'années depuis le dernier cas de rage chez un animal terrestre par rapport à 2016

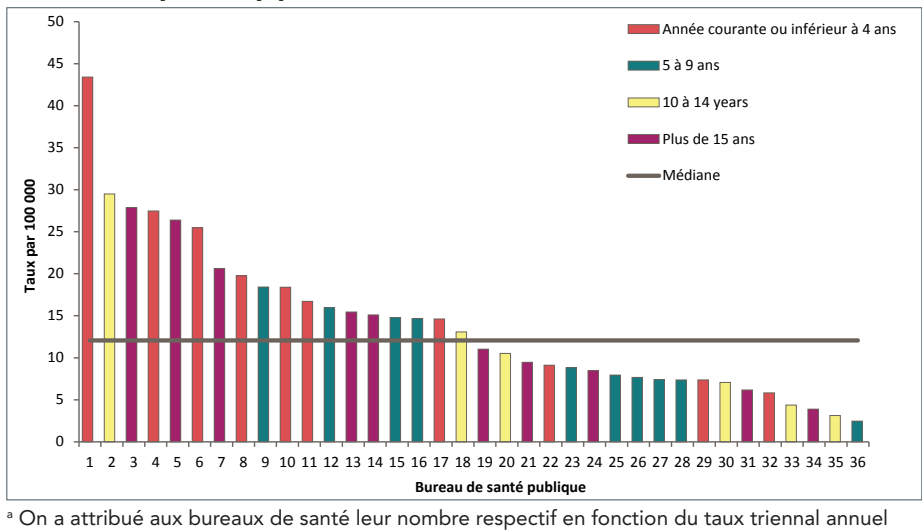

moyen (2014 à 2016) (indiqué à la figure 3), en ordre décroissant

\section{Discussion}

Depuis l'an 2000 environ, le nombre de TPAPE recommandés en Ontario est élevé comparativement au nombre d'animaux enragés, et ce, même lorsqu'on considère l'épizootie dans le centre-ouest de l'Ontario identifiée à la fin de 2015 (figure 2). Depuis 2013, on recommande une évaluation du risque visant à déterminer la nécessité d'un TPAPE lorsqu'une personne est exposée à des chiens, chats ou furets qui n'étaient pas accessibles pour une observation ultérieure. La mise en œuvre d'une évaluation du risque pourrait optimiser le nombre de TPAPE recommandés par les BSP, en particulier au sein des BSP sans cas récent d'animal terrestre enragé.

Les taux annuels de recommandation de TPAPE pour I'exposition à des animaux terrestres de 2014 à 2016 en Ontario variaient de façon nette entre les BSP, soit de 3,0 à 35,2 par 100000 personnes. Cependant, en général, le taux de TPAPE annuel de chaque BSP restait relativement stable au cours des trois années d'étude. Le taux de TPAPE d'un BSP peut être influencé par plusieurs facteurs, dont la probabilité d'exposition humaine à des animaux potentiellement enragés, la probabilité que ces expositions soient signalées au BSP et I'évaluation du risque réalisée par le BSP en collaboration avec le fournisseur de soins de santé. On n'a pas accès à des données permettant d'évaluer la probabilité d'exposition à des animaux potentiellement enragés par BSP, la probabilité de déclaration de ces expositions ou la façon à laquelle on a procédé à l'évaluation du risque. Quoi qu'il en soit, les données sur les cas confirmés d'animaux enragés au sein de la compétence d'un BSP, soit un facteur important à considérer lors d'une évaluation du risque, sont accessibles. Notre analyse a permis de découvrir que la[récence]des derniers animaux terrestres enragés ne semblait pas associée aux taux de recommandation de TPAPE respectifs par BSP. Cinq BSP présentaient des taux de TPAPE pour les animaux terrestres supérieurs à la médiane provinciale, et ce, malgré l'absence de signalement d'un animal terrestre enragé depuis plus de 15 années.

Une explication possible du manque d'association entre les taux de recommandation de TPAPE pour les animaux terrestres et la date du dernier cas de rage chez les animaux terrestres pourrait être qu'au lieu de procéder à une évaluation du risque lorsqu'un animal n'était pas accessible aux fins d'observation, certains BSP recommandaient automatiquement le TPAPE, ce qui est conforme aux recommandations canadiennes et ontariennes avant 2013. Une autre explication possible pour ce manque d'association est que la présence de rage chez des animaux terrestres n'est pas pondérée à grande échelle lorsqu'on évalue le risque de rage après une exposition à des animaux terrestres quand l'animal n'est pas accessible aux fins d'observation.

La possibilité que la rage puisse découler de sources hors du territoire du BSP en raison de l'incursion d'animaux enragés provenant de régions voisines, de l'incursion d'animaux enragés 
transférés ou de l'adoption d'animaux enragés peut influencer la décision de recommander un TPAPE, et ce, même si ces événements sont sporadiques. L'incursion à partir de régions voisines en tant que possible source d'introduction de rage chez des animaux terrestres ne concerne que les BSP à la frontière d'une autre province ou des É.-U. II n'y a pas eu d'épizootie de la rage du raton laveur en Ontario découlant de l'incursion à partir de régions voisines aux États-Unis depuis plus de 10 ans et on n'a jamais pu démontrer sa provenance de provinces voisines (15). Quatre des cinq BSP n'ayant pas connu d'animal enragé sur leur territoire depuis plus de 15 ans et avec des taux de recommandation de TPAPE supérieurs au taux médian ne partagent pas de frontière terrestre avec les États-Unis.

Le transfert d'un animal enragé importé qui entraîne une épizootie non diagnostiquée constitue une préoccupation. Au cours des dernières années, on a connu un épisode diagnostiqué de transfert provenant d'un raton laveur « en visite » qui a provoqué l'épizootie actuelle au centre-ouest de l'Ontario (15). On n'a signalé qu'une fois l'adoption d'un animal domestique enragé en Ontario au cours des dernières années, lorsqu'on a transporté un chiot d'une région rurale de l'Ontario à un marché aux puces de Toronto en 2008. On a constaté la situation avec rapidité et les nombreuses personnes exposées au chiot ont été gérées de manière appropriée. Dans le but d'offrir une protection contre la rage, on doit faire vacciner les chiens et chats de plus de trois mois importés au Canada (avec de petites exceptions) $(16,17)$, quoique des dossiers aient été contrefaits à l'occasion (18-21).

Les chauves-souris constituent une autre source probable d'introduction de la rage chez les animaux terrestres; elles sont connues pour être porteuses de la rage en Ontario. De 2000 à 2018, le typage de souche rabique en Ontario a permis $d$ 'identifier onze animaux terrestres atteints de rage attribuable aux chauves-souris : la mouffette (2001, 2004, 2016, 2018), le chat $(2002,2004)$, le renard $(2003,2009)$, le raton laveur (2002), le bovin (2009) et le chien (2012) (communication personnelle, R. Gagnon, ministère des Richesses naturelles et des Forêts, 20 mars 2018). II n'y a pas de preuve de transmission entre animaux, étant donné le manque de regroupement identifié de tout animal dans le temps. De plus, une recherche documentaire n'a permis de révéler qu'un seul article décrivant la transmission de la rage attribuable aux chauves-souris parmi les animaux terrestres, soit chez la mouffette en Arizona (22). De plus, la transmission de la rage attribuable aux chauves-souris par des animaux terrestres aux humains semble très rare : seuls trois articles, tous d'Amérique du Sud, ont identifié de manière définitive la transmission de la rage attribuable aux chauves-souris à quatre humains par l'entremise de chats (23-25). Il s'agissait de chauves-souris vampires (Desmodus rotundus) qui se nourrissent de sang de mammifères, ce qui accroît la probabilité d'infection chez les animaux terrestres; les chauves-souris de l'Ontario se nourrissent d'insectes.

\section{Limites}

Nos analyses par BSP sont sujettes à un certain nombre de restrictions. Comme on l'a déjà mentionné, nous ne pouvons pas évaluer d'autres facteurs pouvant avoir des conséquences sur les taux de TPAPE, tels que l'ampleur de l'exposition humaine à des animaux terrestres et le signalement ultérieur aux BSP. Souvent, les BSP ne saisissent pas d'information sur les recommandations de TPAPE lorsqu'il n'est pas administré. De plus, il est possible que l'exposition soit survenue à l'extérieur de la frontière géographique du BSP. En ce qui concerne les animaux terrestres enragés, les données se limitent à l'ampleur de la surveillance dans la région particulière. En général, on analyse les animaux pour voir s'ils sont porteurs de la rage en cas d'exposition humaine potentielle ou lors d'épizootie. Par conséquent, on pourrait manquer des animaux terrestres enragés dans le territoire d'un BSP.

Comme autre restriction, notre analyse a pris en considération le moment du dernier animal terrestre enragé, mais pas les taux d'incidence au moment de ce dernier animal enragé (c.-à-d. elle n'a pas considéré s'il y avait un animal enragé par rapport à plusieurs animaux enragés) et nous n'avons pas intégré la date du dernier cas de rage chez les animaux terrestres au sein des BSP voisins dans l'analyse; il s'agit de deux facteurs pouvant influencer l'évaluation du risque.

\section{Conclusion}

II n'y a pas eu de cas de rage humaine acquise d'animaux terrestres depuis plus de 50 ans au Canada. Les lignes directrices canadiennes et ontariennes recommandent de procéder à une évaluation du risque lorsqu'une personne est mordue ou griffée par un chien, un chat ou un furet et que cet animal n'est pas accessible pour une période d'observation ultérieure de 10 jours. La prise en considération du moment de l'animal terrestre enragé le plus récent dans la région constitue un important facteur pour ce qui est de déterminer la nécessité d'un TPAPE lors de l'évaluation du risque.

Les taux d'administration du TPAPE concernant l'exposition à des animaux terrestres par le BSP semblent relativement stables au sein de chaque BSP lorsqu'on les mesure sur une période de trois ans. Parmi les dix BSP sans animal terrestre enragé dans leur région depuis plus de 15 ans, cinq d'entre eux présentaient des taux de recommandation de TPAPE pour l'exposition à des animaux terrestres supérieurs au taux de recommandation de TPAPE médian provincial. Conjuguée à d'autres facteurs, la considération de la présence de rage chez des animaux terrestres sur un territoire peut simplifier l'évaluation du risque concernant les chiens, chats ou furets qui ne sont pas accessibles pour une observation. 


\section{Déclaration des auteurs}

D. M. - Conceptualisation, analyse et interprétation des données, rédaction et révision du document

L. F. - Analyse et interprétation des données, rédaction et révision du document

S. J. - Analyse et interprétation des données, rédaction du document

S. B. - Analyse et interprétation des données, révision du document

B. W. - Conceptualisation, interprétation des données, rédaction et révision du document

\section{Conflit d'intérêts}

Aucun.

\section{Financement}

Le présent travail a reçu l'appui de Santé publique Ontario.

\section{Remerciements}

Nous souhaitons remercier K. Middel et B. Stevenson du ministère des Richesses naturelles et des Forêts pour avoir fourni des données sur la rage animale, ainsi qu'interprété les données et constatations, les bureaux de santé publique de I'Ontario pour avoir saisi des données sur le traitement préventif antirabique postexposition dans le Système d'information sur la santé publique intégré (SISP-i), S. Massarella (Santé publique Ontario) pour avoir entrepris la recherche documentaire, de même que C. Martel (Santé publique Ontario) pour la révision de la traduction.

\section{Références}

1. Stevenson B. Raccoon strain rabies continues to climb. The rabies reporter. MNRF Publication 51709 2016;27(1):1-2. http://www.ontla.on.ca/library/repository/ ser/140213/2016//2016v27no01.pdf

2. Comité consultatif national de l'immunization. Guide canadien d'immunisation: Partie 4 - Agents d'immunisation active: Vaccin contre la rage. Ottawa (ON): Agence de la santé publique du Canada; 2015. https://www.canada. $\mathrm{ca} / \mathrm{fr} /$ sante-publique/services/publications/vie-saine/ guide-canadien-immunisation-partie-4-agents-immunisationactive/page-18-vaccin-contre-rage.html

3. Agence canadienne d'inspection des aliments. Cas de rage au Canada 2018. Ottawa (ON): Gouvernement du Canada. http://www.inspection.gc.ca/animaux/animaux-terrestres/ maladies/declaration-obligatoire/rage/cas-de-rage-aucanada-2018/fra/1550860268670/1550860329289
4. Middleton D, Johnson KO, Rosatte RC, Hobbs JL, Moore SR, Rosella L, Crowcroft NS. Human rabies post-exposure prophylaxis and animal rabies in Ontario, Canada, 20012012. Zoonoses Public Health 2015 Aug;62(5):356-64. DOI PubMed

5. Middleton D, Edwin JJ, Johnson KO, Warshawsky BF. A descriptive analysis of rabies post-exposure prophylaxis data: 2013, Ontario, Canada. Zoonoses Public Health 2018 Feb;65(1):e66-78. DOI PubMed

6. Nunan CP, Tinline RR, Honig JM, Ball DG, Hauschildt P, LeBer CA. Postexposure treatment and animal rabies, Ontario, 1958-2000. Emerg Infect Dis 2002 Feb;8(2):214-7. DOI PubMed

7. Ribadeau Dumas F, N'Diaye DS, Paireau J, Gautret P, Bourhy H, Le Pen C, Yazdanpanah Y. Cost-effectiveness of rabies post-exposure prophylaxis in the context of very low rabies risk: A decision-tree model based on the experience of France. Vaccine 2015 May;33(20):2367-78. DOI PubMed

8. Population and Public Health Division, Ontario Ministry of Health and Long-Term Care. Rabies prevention and control protocol, 2013. Toronto (ON): Ontario Ministry of Health and Long-Term Care; 2013 Sep. 196-202

9. Population and Public Health Division, Ontario Ministry of Health and Long-Term Care. Rabies prevention and control protocol, 2018. Toronto (ON): Ontario Ministry of Health and Long-Term Care; 2018. http://www.health.gov.on.ca/en/ pro/programs/publichealth/oph_standards/docs/protocols_ guidelines/Rabies_Prevention_and_Control_Protocol_2018_ en.pdf

10. Infectious Disease Policy and Programs Unit, Public Health Policy and Programs Branch, Public Health Division, Ontario Ministry of Health and Long-Term Care. Guidance document for the management of suspected rabies exposures. Toronto (ON): Ontario Ministry of Health and Long-Term Care; 2013.

11. National Advisory Committee on Immunization. Canadian Immunization Guide. Part A - Active Vaccines: Rabies vaccine. Ottawa (ON): Public Health Agency of Canada; 2013.

12. Public Health Ontario. iPHIS user guide: Rabies post-exposure prophylaxis. Toronto $(\mathrm{ON})$ : Queen's Printer for Ontario; 2016.

13. IntelliHEALTH Ontario. Population estimates 1986-2015. Toronto (ON): Ontario Ministry of Health and Long-term Care; (Accédé 2017-02-10).

14. IntelliHEALTH Ontario. Population Estimates 2016-2017. Toronto(ON): Ontario Ministry of Health and Long-term Care, (Accédé 2017-02-01).

15. Trewby H, Nadin-Davis SA, Real LA, Biek R. Processes underlying rabies virus incursions across US-Canada border as revealed by whole-genome phylogeography. Emerg Infect Dis 2017 Sep;23(9):1454-61. DOI PubMed 
16. Agence Canadienne d'inspection des aliments. Voyage avec des chiens domestiques au importation de ceux-ci. Ottawa: Gouvernement du Canada. http://inspection. gc.ca/animaux/animaux-terrestres/importation/politiques/ animaux-vivants/animaux-de-compagnie/chiens/ fra/1331876172009/1331876307796

17. Agence Canadienne d'inspection des aliments. Importation ou voyageant avec des chats domestiques - Âgés de trois mois ou plus provenant de pays reconnus exempts de la rage par le Canada. Ottawa: Gouvernement du Canada. http://www.inspection.gc.ca/animaux/ animaux-terrestres/importation/politiques/animaux-vivants/ animaux-de-compagnie/chats/exempts-de-la-rage/ $\mathrm{fra} / 1364954808466 / 1364954913271$

18. Sinclair JR, Wallace RM, Gruszynski K, Freeman MB, Campbell C, Semple S, Innes K, Slavinski S, Palumbo G, Bair-Brake H, Orciari L, Condori RE, Langer A, Carroll DS, Murphy J. Rabies in a dog imported from Egypt with a falsified rabies vaccination certificate--Virginia, 2015. MMWR Morb Mortal Wkly Rep 2015 Dec;64(49):1359-62. DOI PubMed

19. Castrodale L, Walker V, Baldwin J, Hofmann J, Hanlon C. Rabies in a puppy imported from India to the USA, March 2007. Zoonoses Public Health 2008 Oct;55(8-10):427-30. PubMed

20. Centers for Disease Control and Prevention (CDC). Rabies in a dog imported from Iraq--New Jersey, June 2008. MMWR Morb Mortal Wkly Rep 2008 Oct;57(39):1076-8. PubMed
21. Hercules $Y$, Bryant NJ, Wallace RM, Nelson R, Palumbo G, Williams JN, Ocana JM, Shapiro S, Leavitt H, Slavinsk S, Newman A, Crum DA, Joseph BE, Orciari LA, Li Y, Yager P, Condori RE, Stauffer KE, Brown C. Rabies in a dog imported from Egypt - Connecticut, 2017. MMWR Morb Mortal Wkly Rep 2018 Dec;67(50):1388-91. DOI PubMed

22. Wenning K, Bergman DL, Worgess B, Levy C, Ferrat S, Marciniak J, Marciniak J, Rupprecht C, Slate D. Managing an outbreak of big brown bat variant rabies in terrestrial wildlife in Arizona. Conference: Rabies in the Americas 2010;XXI.

23. Paez A, Polo L, Heredia D, Nuñez C, Rodriguez M, Agudelo C, Parra E, Paredes A, Moreno T, Rey G. [An outbreak of human rabies transmitted by a cat in the town of Santander de Quilichao, Colombia, 2008]. Rev Salud Publica (Bogota) 2009 Dec;11(6):931-43. DOl PubMed

24. Bustos Claro MM, Álvarez AAA, Carrascal EJB, Martínez LGA Benigni EM, Beltrán AFP, Ayala JAM. [Encephalitis due to rabies secondary to the bite of a cat infected with a rabies virus of Silvester origen] [in Spanish]. Infectio 2013;17(3):16770. DOI

25. Caicedo Y, Paez A, Kuzmin I, Niezgoda M, Orciari LA, Yager PA, Recuenco S, Franka R, Velasco-Villa A, Willoughby RE Jr. Virology, immunology and pathology of human rabies during treatment. Pediatr Infect Dis J 2015 May;34(5):520-8. DOI PubMed

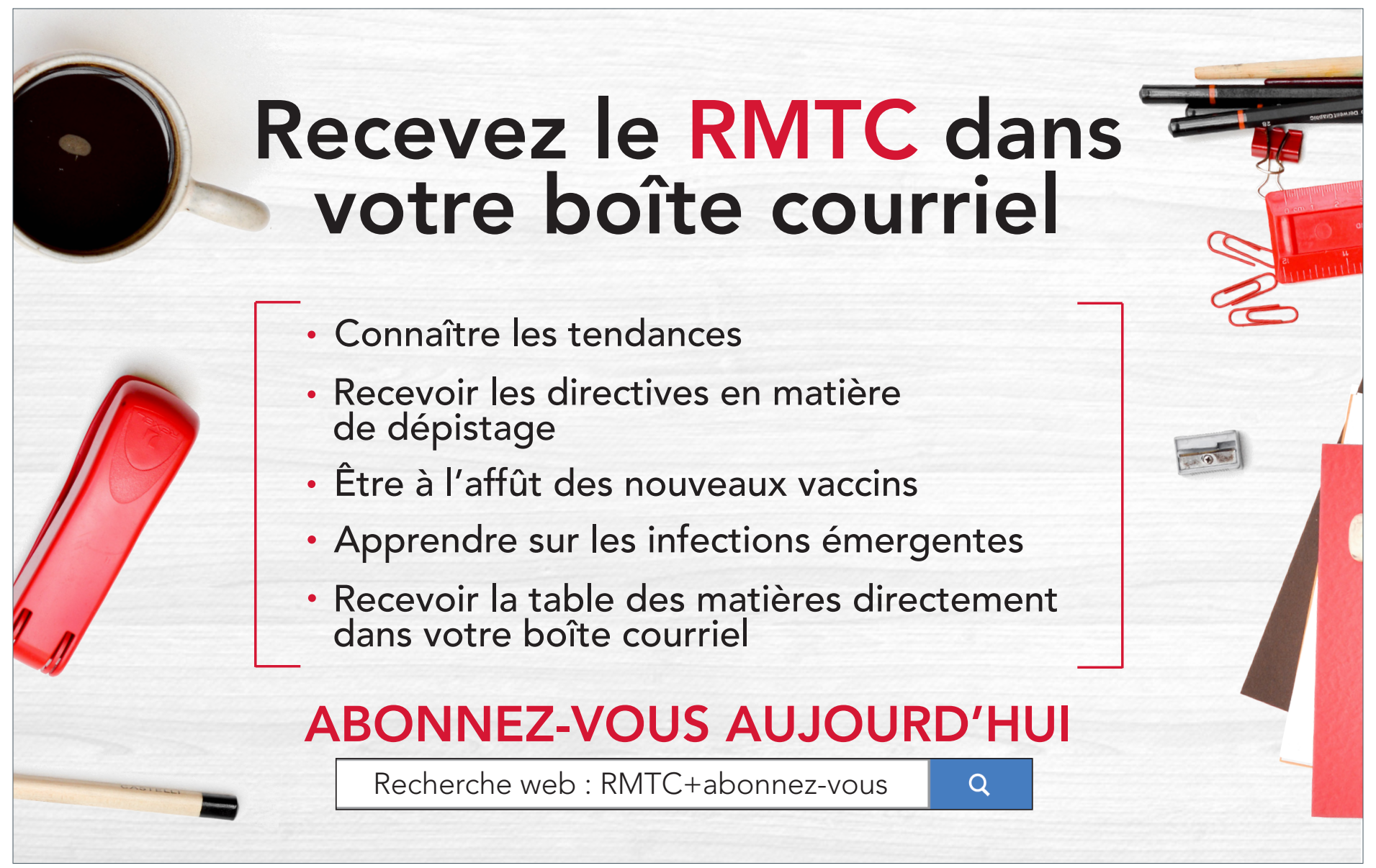

